\title{
Large-Scale Fungicide Spray Heterogeneity and the Regional Spread of Resistant Pathogen Strains
}

\author{
S. Parnell, F. van den Bosch, and C. A. Gilligan
}

First and third authors: Department of Plant Sciences, University of Cambridge, Downing Street, Cambridge CB2 3EA, UK; and first and second authors: Biomathematics, Rothamsted Research, Harpenden, Herts., AL5 2JQ, UK. Accepted for publication 17 January 2006.

\section{ABSTRACT}

Parnell, S., van den Bosch, F., and Gilligan, C. A. 2006. Large-scale fungicide spray heterogeneity and the regional spread of resistant pathogen strains. Phytopathology 96:549-555.

Most models for the spread of fungicide resistance in plant pathogens are focused on within-field dynamics, yet regional invasion depends upon the interactions between field populations. Here, we use a spatially implicit metapopulation model to describe the dynamics of regional spread, in which subpopulations correspond to single fields. We show that the criterion for the regional invasion of pathogens between fields differs from that for invasion within fields. That is, the ability of a fungicide-resistant strain of a pathogen to invade a field population does not necessarily imply an ability to spread through many fields at the regional scale. This depends upon an interaction between the fraction of fields that is sprayed and the reproductive capacity of the pathogen. This result is of practical significance and indicates that resistance management strategies which currently target within-field processes, such as the use of mixtures and alternations of fungicides, may be more effective if between-field processes also were targeted; for example, through the restricted deployment of fungicides over large areas. We also show that the fraction of disease-free fields is maximized when the proportion of fields that is sprayed is just below the threshold for invasion of the resistant strain.

Additional keywords: fitness cost, fungicide effectiveness.
The ability of fungicide-resistant pathogens to spread quickly over large geographical areas has led to increasing problems for the control of plant diseases. A recent example of this is the rapid evolution of resistance to the Strobilurin fungicides in wheat powdery mildew (Blumeria graminis f. sp. tritici) populations in Europe. Strobilurins first were introduced in 1996 to control a range of cereal pathogens; however, by 2000, high frequencies of resistance had been detected over large areas of Germany, France, and the United Kingdom $(8,14)$. The rapid and widespread evolution of resistance on this level highlights the importance of understanding the influence of large-scale processes. However, most studies of fungicide resistance focus on processes at the laboratory or field level and, consequently, the role of large-scale processes such as migration remain poorly understood. Experimentally, large-scale studies are difficult to conduct due to the financial and technical constraints involved. Therefore, modeling is a useful alternative in understanding the dynamics of regionallevel systems where it is not possible to conduct experiments. Surprisingly, most models have not addressed this and have either ignored scale or focused on field-level systems $(12,15,19,22$, 27,28).

Parnell et al. (22) modeled the influence of small-scale heterogeneity in spray coverage (treated and untreated susceptible host tissue) on populations of resistant and sensitive pathogens within a field. Spore migration allows field populations to interact on a regional scale, and high levels of migration have been found for a number of major plant pathogens $(17,18)$. Here, we introduce a spatially implicit metapopulation model to analyze the influence of interactions between field populations under large-scale spray heterogeneity (treated and untreated susceptible fields) on a regional scale. In particular, we are concerned with the factors that

Corresponding author: S. Parnell; E-mail address: sp354@cam.ac.uk

DOI: 10.1094/PHYTO-96-0549

(C) 2006 The American Phytopathological Society influence the invasion, exclusion, and coexistence of resistant and sensitive strains. Thresholds for invasion have been found in previous models, but only for populations within fields $(12,13,19$, 22). Here, we find clear differences at the regional scale compared with field-scale models and, in particular, we show that the regional-scale invasion of resistant strains is determined by a trade-off between the fraction of fields that are sprayed and the intrinsic reproductive ability of the target pathogen.

\section{THEORY AND APPROACHES}

The model. The model describes the population dynamics of a fungal plant pathogen over a large agronomic region comprising a number of fields in which a susceptible host is grown. Fields can be infested with the pathogen, I, or noninfested (i.e., empty), $E$. The following basic version of the model describes the between-field (regional) dynamics of the wild-type pathogen population before the introduction of the fungicide,

$$
\begin{aligned}
& \text { Infested fields: } \mathrm{d} l / \mathrm{d} t=b P I E-\mu l \\
& \text { Empty fields: } \mathrm{d} E / \mathrm{d} t=\mu l-b P I E
\end{aligned}
$$

Infested fields produce spores, which escape the canopy layer and are blown long distances, at rate $P$. Each infested field is assumed to contain an epidemic of equal size and, therefore, produce the same number of spores. Migrating spores land and initiate epidemics within empty fields at rate $b$, and epidemics within fields become extinct at rate $\mu$ due, for example, to the absence of a host as a result of crop rotation. Therefore, the model assumes homogeneous mixing (i.e., a spore originating from one field is equally likely to land on a plant and cause an infection in a neighboring field as in any other field in the system). A summary of the variables and parameters used in the model is given in Table 1.

The basic model is extended to account for the introduction of the fungicide and the arrival of resistance (e.g., by mutation). To 
reflect the heterogeneous deployment of fungicides over regional areas (10), a fraction of fields is assumed to be treated $(\theta)$ and a fraction is untreated $(1-\theta)$. Many plant pathogens evolve a monogenic response to fungicides (29); therefore, we consider two pathogen strains, a sensitive strain and a resistant strain. The main assumptions concerning the within- and between-field dynamics are described below.

Within-field dynamics. The model assumes that, at any one time, individual fields can be infested with either the resistant strain or the sensitive strain but not both. For the purpose of modeling the long-term regional scale dynamics of the resistant strain, the added complexity of within-field coexistence of both strains found in Parnell et al. (22) is not incorporated into the current model. Further, exclusion of either strain following invasion of the other is considered to be instantaneous. These assumptions can be justified in the following way. Models of within-field systems have shown that, when a resistant strain arises in a fungicide-treated field (e.g., through immigration), it will either fail to invade or invade to the exclusion of the sensitive population. The outcome is determined by a trade-off between the effectiveness of the fungicide in reducing the fitness of the sensitive population and the sum of the net fitness costs to the resistant population $(12,13)$. That is, if the fitness cost to the resistant strain is greater than the effectiveness of the fungicide on the sensitive strain, the resistant strain is the inferior competitor and does not invade. However, if the cost to resistance is less than the effectiveness of the fungicide, the resistant strain is the superior competitor and invades to the exclusion of the sensitive strain $(12,13)$.

Where empirical studies have found evidence for a fitness cost to resistance, the cost usually is relatively small $(11,26)$. Conversely, the effectiveness of the fungicide is likely to be high because fungicides with low effectiveness would provide poor disease control and would not be marketable. Therefore, it is likely that the fitness cost to resistance is less than the effectiveness of the fungicide. This implies that the resistant strain is the superior competitor in treated fields and the sensitive strain is the superior competitor in untreated fields. Further, following the invasion of either strain, the time to exclusion of the other strain is likely to be fast relative to the long-term regional dynamics of the population. For example, Bateman et al. (2) showed that carbendazimtreated plots infected with Tapesia spp. (cereal eyespot disease) populations became almost entirely resistant over just one season when inoculated with resistant isolates.

Between-field (regional) dynamics. The addition of the fungicide and the resistant strain to the basic version of the model (equations 1 and 2) yields the full model, which comprises six categories of fields: two categories of empty fields, treated and untreated; and four categories of infested fields, wholly infested with the resistant strain (resistant-infested), wholly infested with the sensitive strain (sensitive-infested), and also either treated or untreated.

The general structure of the full model is the same as the basic version (equations 1 and 2) but the addition of the fungicide and resistant strain incorporates extra complexity in two ways. First, the sporulation $(P)$, infection $(b)$, and extinction rates $(\mu)$ of infested fields are scaled by the cost to resistance $(\rho)$ for resistantinfested fields and the effectiveness of the fungicide $(\varepsilon)$ for sensitive-infested, treated, fields. This yields the following equations for the infested fields,

Treated, sensitive-infested: $\mathrm{d} l_{F S} / \mathrm{d} t=\varepsilon_{b} b\left(\varepsilon_{P} P l_{F S}+P l_{U S}\right) E_{F}-\mu l_{F S}$

Untreated, sensitive-infested: $\mathrm{d} l_{U S} / \mathrm{d} t=b\left(\varepsilon_{P} P l_{F S}+P l_{U S}\right) E_{U}-\mu l_{U S}$

Treated, resistant-infested: $\mathrm{d} l_{F R} / \mathrm{d} t=\rho_{b} b\left(\rho_{P} P l_{F R}+\rho_{P} P l_{U R}\right) E_{F}-\mu l_{F R}$

Untreated, resistant-infested: $\mathrm{d} l_{U R} / \mathrm{d} t=\rho_{b} b\left(\rho_{P} P l_{F R}+\rho_{P} P l_{U R}\right) E_{U}-\mu l_{U R}$

Second, to complete the model system, transitions between resistant-infested and sensitive-infested categories of fields are incorporated into equations 3 to 6 to account for the assumption of within-field exclusion of strains (Figure 1 provides a schematic of these transitions). This yields the full model (equations 7 to 12).

$$
\begin{gathered}
\text { Treated, sensitive-infested: } \\
\mathrm{d} l_{F S} / \mathrm{d} t=\varepsilon_{b} b\left(\varepsilon_{P} P l_{F S}+P l_{U S}\right) E_{F}-\mu l_{F S}-\rho_{b} b\left(\rho_{P} P l_{F R}+\rho_{P} P l_{U R}\right) I_{F S} \\
\text { Untreated, sensitive-infested: } \\
\mathrm{d} l_{U S} / \mathrm{d} t=b\left(\varepsilon_{P} P l_{F S}+P l_{U S}\right) E_{U}-\mu l_{U S}+b\left(\varepsilon_{P} P l_{F S}+P l_{U S}\right) I_{U R} \\
\text { Treated, resistant-infested: } \\
\mathrm{d} l_{F R} / \mathrm{d} t=\rho_{b} b\left(\rho_{P} P l_{F R}+\rho_{P} P l_{U R}\right) E_{F}-\mu l_{F R}+\rho_{b} b\left(\rho_{P} P l_{F R}+\rho_{P} P l_{U R}\right) I_{F S} \\
\text { Untreated, resistant-infested: } \\
\mathrm{d} l_{U R} / \mathrm{d} t=\rho_{b} b\left(\rho_{P} P l_{F R}+\rho_{P} P l_{U R}\right) E_{U}-\mu l_{U R}-b\left(\varepsilon_{P} P l_{F S}+P l_{U S}\right) I_{U R}
\end{gathered}
$$

Empty treated and untreated fields increase and decrease inversely proportional to the fraction of infected fields as in equation 2 and, therefore, in the full version of the model are described by equations 11 and 12 .

$$
\begin{gathered}
\text { Treated, empty: } \\
\mathrm{d} E_{F} / \mathrm{d} t=\mu\left(I_{F S}+I_{F R}\right)-\varepsilon_{b} b\left(\varepsilon_{P} P l_{F S}+P l_{U S}\right) E_{F}-\rho_{b} b\left(\rho_{P} P l_{F R}+\rho_{P} P l_{U R}\right) E_{F} \\
\\
\text { Untreated, empty: } \\
\mathrm{d} E_{U} / \mathrm{d} t=\mu\left(I_{U S}+I_{U R}\right)-b\left(\varepsilon_{P} P l_{F S}+P l_{U S}\right) E_{U}-\rho_{b} b\left(\rho_{P} P l_{F R}+\rho_{P} P l_{U R}\right) E_{U}
\end{gathered}
$$

Methods of analysis. We use a combination of linear stability analysis and model simulations to investigate the dynamics of the model. Linear stability analysis is a well-defined mathematical procedure (9) and allows for the calculation of criteria for the invasion of infected fields into the regional system. The analysis involves, first, assuming that within-field infestations of only one pathogen strain are present in the regional population. The parameter values that allow the other strain to invade then can be calculated. Thus, we determine, on a regional scale, for what parameter combinations either strain is able to invade or become excluded from the system and also for what parameter combinations both strains can invade, indicating coexistence (for a full explanation of the linear stability procedure, see 22). This analysis is corroborated by simulations of the model which are also used to determine the final proportion of different field types (noninfested, resistant-infested, and sensitive-infested) for certain parameter combinations.

TABLE 1. Model variables and parameters

\begin{tabular}{ll}
\hline Symbol & \multicolumn{1}{c}{ Description } \\
\hline Variable & Proportion of noninfested fields with treatment status $i(F=$ treated, $U=$ untreated $)$ \\
$E_{i}$ & Proportion of infested fields with treatment status $i(F=$ treated, $U=$ untreated $)$ and resistance status $j(S=$ sensitive, $R=$ resistant $)$ \\
$I_{i j}$ & Fraction of fields sprayed \\
Parameter & Probability that a spore is deposited in a field and initiates an epidemic \\
$\theta$ & Rate of spore production and escape from infested fields \\
$b$ & Local field extinction rate \\
$P$ & Proportionate reduction in resistant pathogen parameter $i$ to account for the fitness cost to resistance; $0-1$ \\
$\mu$ & Proportionate reduction in pathogen parameter $i$ to account for the fungicide effectiveness; $0-1$ \\
$\rho_{i}$ &
\end{tabular}




\section{RESULTS}

The results from the linear stability analysis and simulations of the model show that the model dynamics are driven by three main components: the relative competitive abilities of the pathogen strains (determined by the fitness cost to resistance, $\rho$, and the effectiveness of the fungicide, $\varepsilon$ ), the fraction of fields sprayed $(\theta)$, and the basic reproductive number of the pathogen for between field infestations, $R_{0}$. $R_{0}$ is a well-known parameter used in invasion biology to summarize the reproductive potential of an organism (1). Here, $R_{0}=b P / \mu$. Therefore, it can be thought of as the average number of new infested fields produced from one previously infested field in an otherwise noninfested landscape. Therefore, $R_{0}$ refers to the intrinsic infectiousness of the wild-type pathogen population before the use of the fungicide, including the effect of prevailing crop management techniques.

Thresholds for invasion, exclusion, and coexistence. The parameters for cost to resistance $(\rho)$ and fungicide effectiveness $(\varepsilon)$ are restricted to low and high parameter ranges, respectively, to reflect the main model assumption of instantaneous withinfield mutual exclusion of resistant and sensitive strains. The linear stability analysis reveals that invasion, exclusion, and coexistence of strains is determined by the direct trade-off between the fraction of fields sprayed $(\theta)$ and the basic reproductive number of infested fields $\left(R_{0}\right)$ (Fig. 2) (the full expressions for the invasion of each pathogen strain are given in Appendix I). The population can evolve to one of four outcomes: sensitive-infested fields only, resistant-infested fields only, coexistence of both field types, or extinction of the pathogen population (Fig. 2). Each of these outcomes is separated in parameter space by a number of thresholds. There also is a critical threshold of the fraction of fields sprayed, denoted as $\theta^{*}$, above which resistant-infested fields always can invade the population and cannot be excluded, and below which resistant-infested fields always cannot invade and can be excluded (Fig. 2). The range of parameter values for mutual exclusion of both strains decreases as $R_{0}$ increases, which permits coexistence (Fig. 2). Extinction of the pathogen population is possible where $R_{0}$ is very low (Fig. 2). An increase in the effectiveness of the fungicide $(\varepsilon)$ decreases $\theta^{*}$ (Fig. 3A and $\mathrm{B}$ ) and an increase in the fitness cost to resistance $(\rho)$ increases $\theta^{*}$ (Fig. 3C and D). Changes in the cost to resistance have a larger influence on $\theta^{*}$ than equal changes in the effectiveness of the fungicide (Fig. 3).

Simulations of the proportion of infested and noninfested fields. The results of the model simulations reveal the influence of the fraction of fields sprayed $(\theta)$ on the final proportions of infested and noninfested fields (Fig. 4A and B) for transects depicted on Figure 1. When $\theta$ is low, the regional population is composed of sensitive-infested fields only. As $\theta$ increases, the proportion of sensitive-infested fields decreases (Fig. 4A and B). Resistant-infested fields can invade the regional system once a higher value of $\theta$ is achieved. Paradoxically, increases in $\theta$ beyond

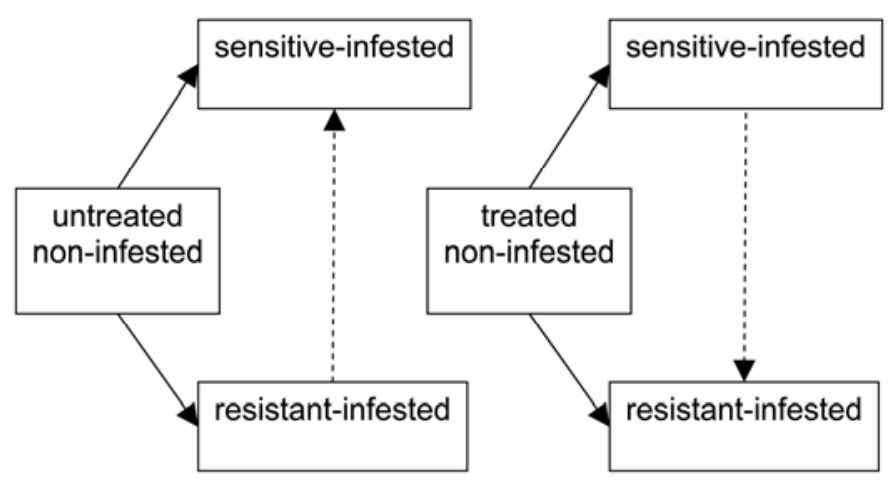

Fig. 1. Schematic depicting the possible transitions of infested and noninfested field types in the model. this point lead to decreases in the total proportion of noninfested fields (Fig. 4A and B). This occurs because increases in $\theta$ select for increases in resistant-infested fields to a greater extent than for decreases in sensitive-infested fields. For higher values of $\theta$, sensitive-infested fields are excluded and the proportion of resistant-infested fields is independent of $\theta$ (Fig. 4A and B). Consequently, the total proportion of infested fields reaches a minimum at the point when resistant-infested fields invade (Fig. $4 \mathrm{~A}$ and $\mathrm{B})$. Therefore, maximum regional control of the pathogen is achieved in the model when as high as possible a fraction of fields is sprayed but below that which permits the invasion of the resistant strain. Increased values of $R_{0}$ result in increases in the total proportion of infested fields, as would be expected intuitively (Fig. 4A and B). The results also show how the equilibrium fraction of resistant- and sensitive-infested fields changes with different fractions of spray coverage, $\theta$ (Fig. 5). As intuition would expect, higher fractions of spray coverage increase the fraction of resistant-infested fields relative to sensitive-infested (Fig. 5).

\section{DISCUSSION}

Using a spatially implicit metapopulation model, we have identified a number of thresholds that determine the long-term regional-scale outcome of a pathogen population following the widespread use of a fungicide. Other than the extreme case of extinction, three outcomes are possible: resistant-infested fields only, sensitive-infested fields only, or coexistence of both. Similar outcomes were found by Parnell et al. (22) for the invasion of resistant strains within a field-scale system. However, we find a number of differences in the current between-field model from those found by Parnell et al. (22). First, the current model shows that high rates of spore production and infection critically influence the thresholds for invasion and coexistence (through $R_{0}$ ). In contrast, spore production and infection rates were shown to have no influence on the thresholds for invasion and coexistence in the within-field model by Parnell et al. (22). Second, Parnell et al.

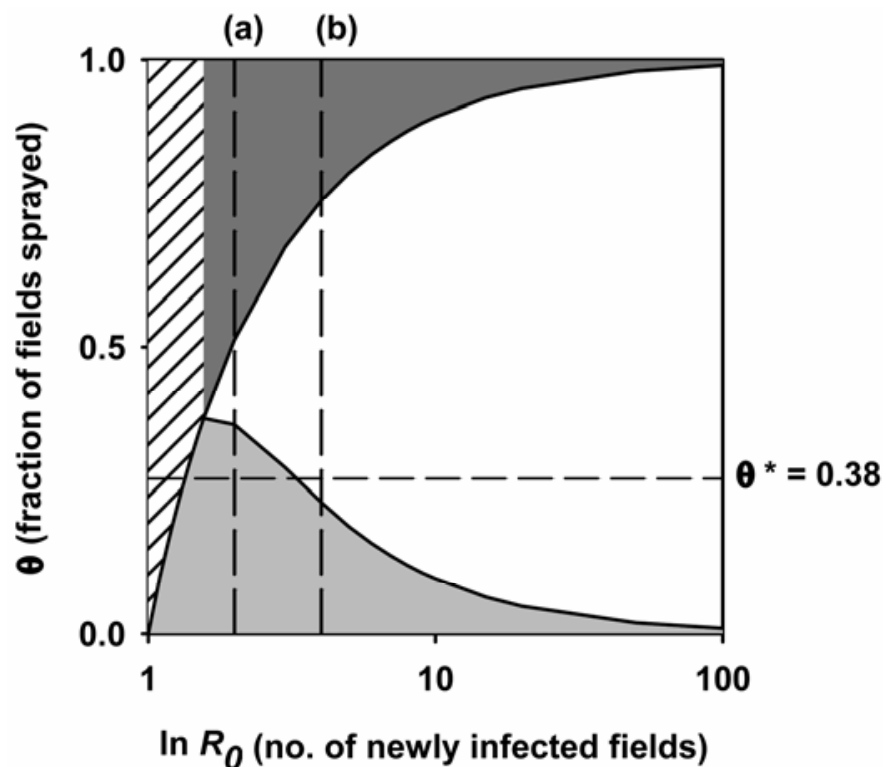

Fig. 2. Thresholds for the invasion, exclusion, and coexistence of resistant and sensitive local populations with dependence on the fraction of fields sprayed, $\theta$, and the reproductive number of infested fields, $R_{0}$. Areas shaded dark gray indicate where the resistant strain takes over all fields, light gray areas indicate where sensitive strains take over all fields, white areas indicate where withinfield populations of both strains coexist, and the shaded area is where both strains are excluded. Lines (a) and (b) refer to transects used for the simulations in Figure 4A and B, respectively. Defaults parameter values are $\varepsilon=0.2$, $\rho=0.8$. 
(22) showed that, when there were high rates of movement between treated and untreated host tissue (high spore dispersal), the parameter region for coexistence decreased. In contrast, we find here that, when the movement between treated and untreated fields is high (high $R_{0}$ ), the parameter region for coexistence increases.

The differences in the results between the models are reflected in the differences in the nature of the host at each scale. That is, in the field-scale model by Parnell et al. (22), the host is represented by susceptible host tissue, whereas in the current model, it is represented by susceptible host fields. The biological relevance is that once host tissue (within-field scale model) becomes infected with either strain, it cannot subsequently become infected with the other strain, whereas this transition can occur in individual field populations. Although dissimilarities in the interpretation of parameters between the two models prevents a closer comparison than this, differences in results between the two scales highlight the fact that the ability of the resistant strain to invade within an individual field does not guarantee invasion between fields on a regional scale. However, there also are similarities between the two scales; for example, decreased spray coverage resulted in a decreased rate of build-up of resistant populations
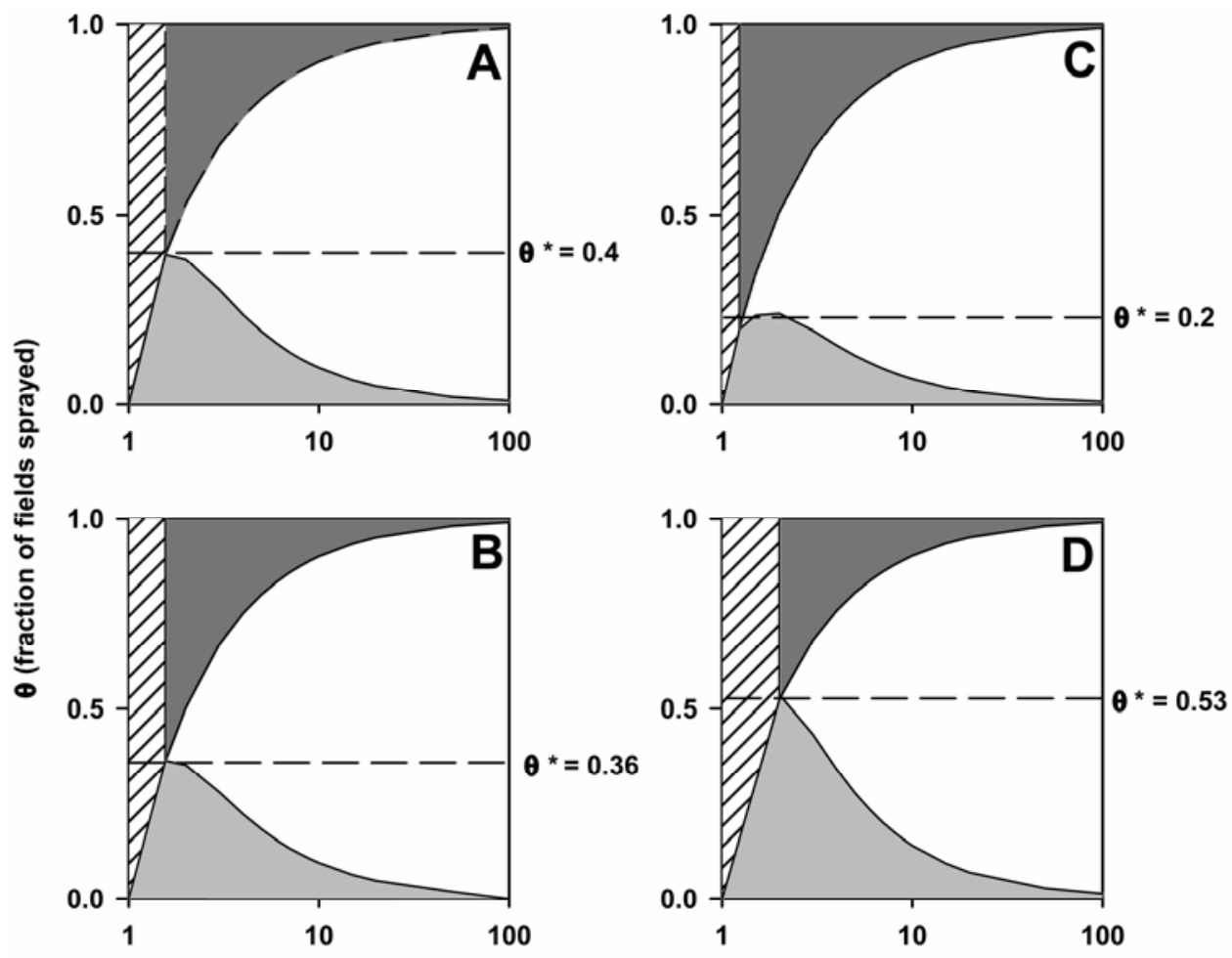

In $R O$ (no. of newly infected fields)

Fig. 3. Thresholds for the invasion, exclusion, and coexistence of resistant and sensitive local populations illustrating the influence of increasing and decreasing the effectiveness of the fungicide, $\varepsilon(\mathbf{A}, \varepsilon=0.3$ and $\mathbf{B}, \varepsilon=0.1)$ and increasing and decreasing the cost to resistance, $\rho(\mathbf{C}, \rho=0.3$ and $\mathbf{D}, \rho=0.1)$. Areas shaded dark gray indicate where the resistant strain takes over all fields, light gray areas indicate where sensitive strains take over all fields, white areas indicate where within-field populations of both strains coexist, and the shaded area is where both strains are excluded.

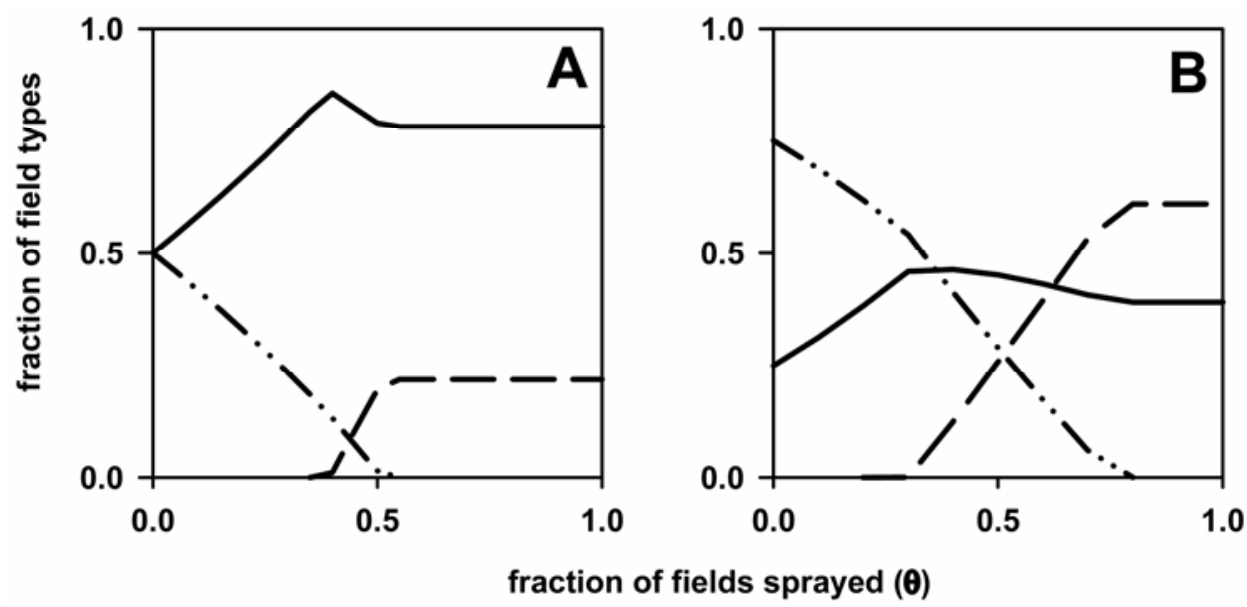

Fig. 4. Change in the fractions of noninfested and infested fields for the fraction of fields sprayed, $\theta$. Noninfested fields are indicated by the solid line, resistantinfested fields are indicated by the dash-dot line, and sensitive-infested fields are indicated by the dashed line. $\mathbf{A}, R_{0}=4$ and $\mathbf{B}, R_{0}=2$. Default parameters are $\varepsilon=$ 0.2 and $\rho=0.8$ 
in both the current model (Fig. 5) and in the within-field model by Parnell et al. (22) (detailed analyses are given in literature citation 21).

Previous models of fungicide resistance either have not focused on heterogeneity in spray coverage or have included spray heterogeneity but have focused on field-scale processes or largely ignored the effects of scale $(7,12,15,19,27,28)$. However, an earlier study by Milgroom et al. (20) introduced a density-independent migration model for the evolution and spread of resistance in a population consisting of sprayed and unsprayed fields. Milgroom et al. (20) found that the resistant strain could dominate all fields in a region if the migration rate between fields was sufficiently high to dilute the advantage the sensitive strain has in untreated fields. Here, we find that the competitive outcome also depends critically on the fraction of fields that are sprayed. In addition, we find that, if the between-field movement of the pathogen is high (high $R_{0}$ ), the resistant strain will dominate all treated fields but the sensitive strain will dominate all untreated fields. This can be explained as follows: in the long term, resistant strains are competitively superior in treated fields and sensitive strains are competitively superior in untreated fields. If $R_{0}$ is very high, mixing is complete and all treated fields become infested with the resistant strain and all untreated fields become infested with the sensitive strain. However, if $R_{0}$ is low, strains cannot move between fields to the extent to allow them to capitalize on their within-field competitive advantage and, therefore, may be excluded. The outcome then is dependent on the fraction of fields sprayed; for example, if few enough fields are treated (below $\theta^{*}$ ) (Fig. 2) resistant-infested fields cannot invade.
Similar studies in insecticide resistance have focused on the high-dose refuge strategy $(6,25)$. This two-pronged strategy involves the use of high doses in treated areas to kill off heterozygous resistant individuals, and the incorporation of refuges of untreated areas to increase the relative fitness of the sensitive population. In the current article, we consider only a haploid system and, therefore, only the refuge element of this strategy. These models generally agree with the results presented here (Fig. 4) and show that an increase in movement of the insect leads to an increase in the frequency of resistance $(6,23,24)$; however, some results are confounded by the incorporation of other interacting factors, such as crop rotation $(23,24)$. In addition, one study also reports a critical size of untreated area below which insecticide-resistant individuals cannot invade (16). The authors (16) assumed that resistant individuals carried a fitness cost and noted a caveat also relevant to the current model. That is, the possibility of the evolution of fitness modifiers that reduce the cost of resistance and, thus, the effectiveness of a refuge strategy to prevent invasion of resistant strains. This process, termed "compensatory evolution," has been reported in some studies of fungicide resistance $(3,31)$ and shown to be theoretically possible if "compensatory" genes are available in the population (30).

The model is spatially implicit and assumes homogenous mixing of spores within the system. The transition of the model to a spatially explicit formulation most likely will lead to further insights. For example, metapopulation theory indicates that global dispersal is more efficient at balancing extinction rates in metapopulations than local dispersal. Therefore, for pathogens with
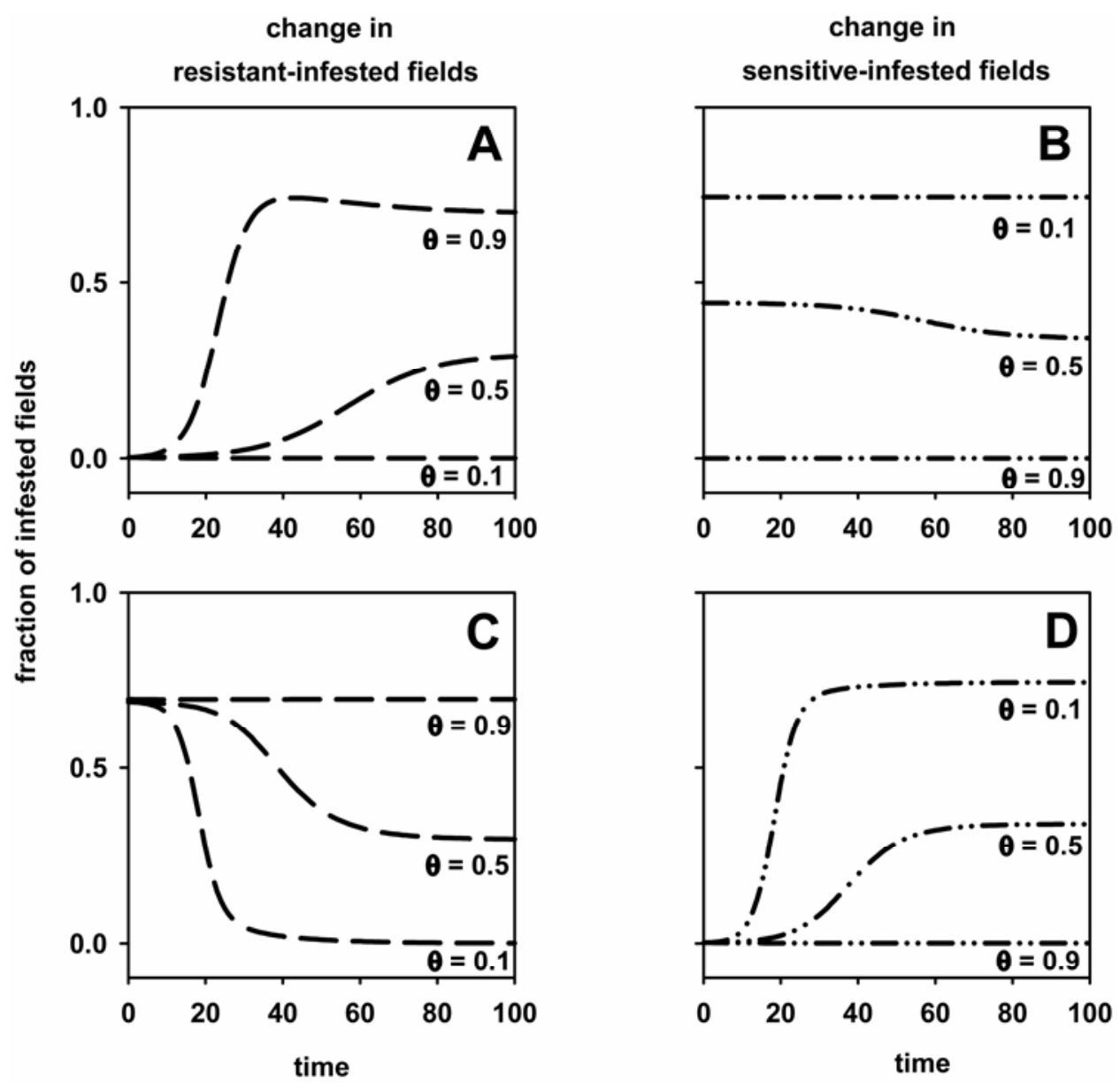

Fig. 5. Change with time in the fractions of $\mathbf{A}$ and $\mathbf{C}$, resistant- and $\mathbf{B}$ and $\mathbf{D}$, sensitive-infested fields for different fractions of spray coverage, $\theta$. Plots $\mathbf{A}$ and $\mathbf{B}$ (horizontal top) show the scenario of the resistant strain invading a system consisting of sensitive strain only and in steady state. Plots $\mathbf{C}$ and $\mathbf{D}$ (horizontal bottom) show the scenario of the sensitive strain invading a system consisting of the resistant strain only and in steady state. 
short-range dispersal capabilities, it is likely that we underestimate the fraction of treated area required for the resistant strain to invade. Similarly, the spatial pattern of treated fields will influence the invasion of the resistant strain. Using a spatially explicit simulation model, Peck (24) showed that, where treated fields were clumped, the intensity of selection was sufficient to generate the development of resistance. A spatially explicit modeling approach also will lead to important insights regarding the differential spatiotemporal effects of mutation and migration. For example, in pathogens with predominantly short-range dispersal capacities, such as eyespot disease of cereals (Oculimacula acuformis and $O$. yallundae), resistance can be expected to spread from single foci if the mutation rate toward resistance is low, but from multiple foci if the mutation rate is high.

Concluding remarks. The ability of major plant pathogens to disperse spores over large geographical areas allows local pathogen field populations to interact over regional areas. The results presented here indicate that long-range spore dispersal is an important consideration in the development of fungicide resistance, though we have not explicitly modeled this. The model has shown that there are differences between the factors that influence invasion in within-field systems from between-field systems (regional scale) and we have identified specific criteria for the regional scale invasion of resistant populations. This indicates that resistance management strategies that currently focus on within-field processes (for example, the use of mixtures and alternations) (4) may be more effective if between-field processes were targeted also (for example, the restricted deployment of fungicides over large areas). This could prevent the invasion of the resistant strain in the long term (Fig. 2). The model highlights the importance of preventing the invasion of resistant populations by showing that the minimum fraction of infested fields is achieved before they invade (Fig. 4). Further work should investigate the practical significance of these results as they relate to equilibrium conditions which may not be reached in the agricultural systems described. Failing this, however, reduced spray coverage will at least reduce the rate of build-up of resistant-infested fields (Fig. 5). Implementing regional-scale strategies may be practically more challenging than implementing recommendations at field scale; however, the United States Environmental Protection Agency (EPA) has shown how regional-level regulations can be used to manage insect resistance to Bt cotton (5). From 1996 to 2000, each grower in the state of Arizona was required to ensure that a specific percentage of their cotton acreage was planted with non-Bt cotton. It is easy to see how similar farm-level regulation could be implemented with regard to the restricted use of fungicides. Disease control then can be maintained in untreated fields through the use of separate control methods; for example, cultural control or the use of fungicides with distinct modes of action (i.e., not exhibiting cross resistance).

This work has provided an insight into the importance of the influence of regional-level processes such as spore migration on the evolution of resistant strains. In addition to the limitation imposed by the possibility of compensatory evolution, the model also does not incorporate interruptions in cropping (i.e., seasonality) due to harvest. Future work will investigate the role of these processes on the regional-scale dynamics of resistant strains and also the role of different spatial patterns in the deployment of treated areas, the effects of aggregated spore dispersal, and the directional movement of spores.

\section{APPENDIX I}

The invasion criteria. To determine the criteria for the invasion of either pathogen strain into a steady-state system containing only the other strain, a stability analysis of the model was conducted. This technique is identical to that done in Parnell et al. (22; Appendix II), to which the reader is referred.
The sensitive strain can invade when

$\Omega-\mathrm{K}<0$

where

$$
\begin{aligned}
& \Omega=(1-\varepsilon)^{2} \mathrm{bPA}-(1-\rho) \mathrm{b}\left(\frac{(\theta \mathrm{C}-\mu)-(\theta-1)}{\mathrm{C}}-\mu\right)\left(\mathrm{bp}\left(1-\theta+\frac{\mathrm{B}}{\mathrm{bp}}\right)\right) \\
& \mathrm{K}=-(1-\varepsilon) \mathrm{bPA}(1-\varepsilon) \mathrm{bP}((1-\theta)+\mathrm{B})(1-\varepsilon) \mathrm{bP}\left(1-\theta+\frac{\mathrm{B}}{\mathrm{bp}}\right)-\mathrm{B}-\mu
\end{aligned}
$$

and

$\mathrm{A}=\left(\theta-\frac{\left(\theta(1-\rho)^{2} \mathrm{bP}-\mu\right)}{(1-\rho)^{2} \mathrm{bp}}\right)$

$\mathrm{B}=\frac{\left(\theta(1-\rho)^{2} \mathrm{bP}-\mu\right)(\theta-1)}{(1-\rho)^{2}}$

$\mathrm{C}=(1-\rho)^{2} \mathrm{bP}$

The resistant strain can invade when

$\Psi-\Phi<0$

where

$\Psi=(\mathrm{C} \theta-\mu)\left(\mathrm{C}(1-\theta)-\frac{(\mathrm{D}+\mathrm{E}) \mathrm{bP}(\mathrm{C}-1)}{2((1-\varepsilon) \mathrm{bp}-\mathrm{bp})}-\frac{(1-\varepsilon) \mathrm{bP}(\mathrm{F}-\mathrm{E})}{2\left((1-\varepsilon)^{3} \mathrm{bp}-(1-\varepsilon)^{2} \mathrm{bp}\right)}-\mu\right)$

$\Phi=-\mathrm{C} \theta\left(\mathrm{C}(1-\theta)-\frac{\mathrm{C}(\mathrm{F}-\mathrm{E})}{2\left((1-\varepsilon)^{3} \mathrm{bp}-(1-\varepsilon)^{2} \mathrm{bp}\right)}\right)$

and

$\mathrm{C}=(1-\rho)^{2} \mathrm{bP}$

$\mathrm{D}=\mathrm{bp}\left(2 \theta-(1-\varepsilon) \theta-2-(1-\varepsilon)-(1-\varepsilon)^{2} \theta\right)+\mu(1-\varepsilon)$

$(1-\varepsilon)^{4} \mathrm{~b}^{2} \mathrm{P}^{2} \theta^{2}-2(1-\varepsilon)^{3} \mathrm{~b}^{2} \mathrm{P}^{2} \theta^{2}-2(1-\varepsilon)^{2} \mathrm{~b}^{2} \mathrm{P}^{2} \theta+(1-\varepsilon)^{2} \mathrm{~b}^{2} \mathrm{P}^{2} \theta^{2}$

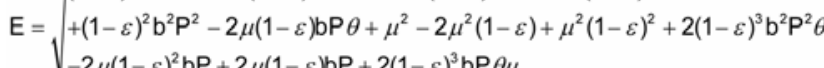

$-2 \mu(1-\varepsilon)^{2} \mathrm{bP}+2 \mu(1-\varepsilon) \mathrm{bP}+2(1-\varepsilon)^{3} \mathrm{bP} \theta \mu$

$\mathrm{F}=\mathrm{bp}\left(2 \theta(1-\varepsilon)^{3}-(1-\varepsilon)^{2} \theta+(1-\varepsilon)-(1-\varepsilon) \theta\right)+\mu(1-\varepsilon)$

\section{ACKNOWLEDGMENTS}

This work was funded by the Biotechnology and Biological Research Council (BBSRC).

\section{LITERATURE CITED}

1. Anderson, R., and May, R. 1979. Population biology of infectious diseases. Part I. Nature 280:361-367.

2. Bateman, G. 1990. Comparison of the effects of prochloraz and flusilazole on foot rot diseases and on populations of the eyespot fungus, Pseudocercosporella herpotrichoides, in winter wheat. Z. Pflanzenkrankh. Pflanzenpathol. Pflanzenschutz 97:508-516.

3. Bierman, S., Fitt, B., van den Bosch, F., Bateman, G., Jenkyn, J., and Welham, S. 2002. Changes in populations of Tapesia yallundae and Tapesia acuformis (eyespot) under different fungicide regimes in successive crops of winter wheat, 1984-2000. Plant Pathol. 51:191-201.

4. Brent, K. 1995. Fungicide resistance in crop pathogens: How can it be managed? Fungicide Resistance Action Committee Monograph No. 1. Fungicide Resistance Action Committee, Global Crop Protection Federation, Brussels.

5. Carriere, Y., Dennehy, T., Pedersen, B., Haller, S., Ellers-Kirk, C., Antilla, L., Liu, Y., Willott, E., and Tabashnik, B. 2001. Large-scale management of insecticide resistance to transgenic cotton in Arizona: Can transgenic insecticidal crops be sustained? J. Econ. Entomol. 94:315-325.

6. Cerda, H., and Wright, D. 2004. Modelling the spatial and temporal location of refugia to manage resistance in Bt transgenic crops. Agric. Ecosyst. Environ. 102:163-174.

7. Chin, K. 1987. A simple model of selection for fungicide resistance in plant pathogen populations. Phytopathology 77:666-669.

8. Chin, K., Chavaillaz, D., Kaesbohrer, M., Staub, T., and Felsenstein, F. 2001. Characterising resistance risk of Erysiphe graminis f. sp. tritici to strobilurins. Crop Prot. 20:87-96.

9. Edelstein-Keshet, L. 1988. Mathematical Models in Biology. McGrawHill Science, Boston. 
10. Garthwaite, D., and Thomas, M. 2000. Pesticide Usage Survey Report: Arable Crops in Great Britain. Department of the Environment, Food and Rural Affairs, York, UK.

11. Gisi, U., Sierotzki, H., Cook, A., and McCaffery, A. 2002. Mechanisms influencing the evolution of resistance to Q0 inhibitor fungicides. Pest Manage. Sci. 58:859-867.

12. Gubbins, S., and Gilligan, C. 1999. Invasion thresholds for fungicide resistance: Deterministic and stochastic analyses. Proc. R. Soc. Lond. Ser. B 266:2539-2549.

13. Hall, R., Gubbins, S., and Gilligan, C. 2004. Invasion of drug and pesticide resistance is determined by a trade-off between treatment efficacy and relative fitness. Bull. Math. Biol. 66:825-840.

14. Heaney, S., Hall, A., Davies, S., and Olaya, G. 2000. Resistance to fungicides in the Qol-STAR cross-resistance group: Current perspectives. Proc. 2000 BCPC Conf. Pests Dis. 2:755-762.

15. Kable, P., and Jeffrey, H. 1980. Selection for tolerance in organisms exposed to sprays of biocide mixtures: A theoretical model. Phytopathology 70:8-12.

16. Lenormand, T., and Raymond, M. 1998. Resistance management: The stable zone strategy. Proc. R. Soc. Lond. Ser. B 265:1985-1990.

17. Limpert, E. 1999. Dispersal of cereal mildews across Europe. Agric. For. Meteorol. 97:293-308.

18. Linde, C., Zhan, J., and McDonald, B. 2002. Population structure of Mycosphaerella graminicola: From lesions to continents. Phytopathology 92:946-955.

19. Milgroom, M. 1990. A stochastic model for the initial occurrence and development of fungicide resistance in plant pathogen populations. Phytopathology 80:410-416.

20. Milgroom, M., Levin, S., and Fry, W. 1989. Population genetics theory and fungicide resistance. Pages 340-367 in: Plant Disease Epidemiology.
K. Leonard and W. Fry, eds. McGraw Hill, New York.

21. Parnell, S. 2005. Page 179 in: The Invasion and Spread of Fungicide Resistance. Department of Plant Sciences, Ph.D. thesis, University of Cambridge, Cambridge.

22. Parnell, S., Gilligan, C., and van den Bosch, F. 2005. Small-scale fungicide spray heterogeneity and the coexistence of resistant and sensitive pathogen strains. Phytopathology 95:632-639.

23. Peck, S., and Ellner, S. 1997. The effect of economic thresholds and lifehistory parameters on the evolution of pesticide resistance in a regional setting. Am. Nat. 149:43-63.

24. Peck, S., Gould, F., and Ellner, S. 1999. Spread of resistance in spatially extended regions of transgenic cotton: Implications for management of Heliothis virescens (Lepidoptera: Noctuidae). J. Econ. Entomol. 92:1-16.

25. Rausher, M. 2001. Co-evolution and plant resistance to natural enemies. Nature 411:857-864.

26. Schmidt, C. 1995. Research on Propiconazole sensitivity in Septorianodorum Berk. in a long term field monitoring. Z. Pflanzenkrankh. Pflanzenpathol. Pflanzenschutz 102:375-391.

27. Shaw, M. 1989. A model of the evolution of polygenically controlled fungicide resistance. Plant Pathol. 38:44-55.

28. Shaw, M. 2000. Models of the effects of dose heterogeneity and escape on selection pressure for pesticide resistance. Phytopathology 90:333-339.

29. Skylakakis, G. 1985. Two different processes for the selection of fungicide-resistant sub-populations. OEPP/EPPO Bull. 15:519-525.

30. Wijngaarden, P., van den Bosch, F., Jeger, M., and Hoeskstra, R. 2005. Adaptation to the cost of resistance: A model of compensation, recombination, and selection in a haploid organism. Proc. R. Soc. Lond. Ser. B 272:85-89.

31. Wolfe, M. 1985. Dynamics of the response of barley mildew to the use of sterol synthesis inhibitors. OEPP/EPPO Bull. 15:451-457. 